\title{
Metronomic Chemotherapy in Selected Children with Refractory Tumors; Report of Four Cases and a Review of the Literature
}

\author{
Mahdi Shahriari ${ }^{1}$ and Babak Abdolkarimi ${ }^{2 *}$ \\ ${ }^{1}$ Department of Pediatric Hematology Oncology, Shiraz University of Medical Sciences, Iran \\ ${ }^{2}$ Department of Pediatric Hematology Oncology, Lorestan university of Medical Sciences, Iran
}

*Corresponding author: Babak Abdolkarimi, Asistant professor of pediatric hematology oncology. Lorestan university of Medical Sciences, khorramabad, Iran.
Received Date: May 25, 2019

Published Date: May 30, 2019

\section{Introduction}

Metronomic chemotherapy is the continual, systemic administration of non-toxic chemotherapy doses that target proliferating endothelial cells during tumor angiogenesis [1]. This strategy began 40 years ago in adult oncology, but there is few experiences in pediatric settings [2]. After a systematic review on evidence-based documents and selection of metronomic chemotherapy protocols from the literature; eligible patients were received this kind of chemotherapy after informed consent taken from their parents.

\section{Case Representation}

\section{Case 1}

A 4 years old girl with sage IV Neuroblastoma (N-Myc Amplification was negative) who received conventional chemotherapy after surgery and resection of her left adrenal mass, but her Bone Marrow (BM) involvement remained, so intensive chemotherapy with ICE Protocol (Ifosfamide, carboplatin and Etoposide) repeated for 6 courses; but against stage 3-4 neutropenia and mucositis, her BM still had 25\% Neuroblasts. At this time MIBG Scan was diffusely positive in the left side of abdomen and BM. She had no donor for allogenic BM transplant and parents refused from Autologous BM transplantation due to its risks and prolonged hospital stay. She was put on metronomic chemotherapy, and after 3 courses her MIBG showed improvement (only some BM involvement). Now she is alive and active and after 5th course Cyclophosphamide and Methotrexate was omitted.

Case 2

A 12 years old girl with Right Adrenal mass was diagnosed a mixed tumor composed of 60\% Neuroblastoma, 20\% Ganglio neuroblastoma and 20\% Ganglioneuroma. Resection of tumor was incompletely done due to encasement of Abdominal Aorta and Inferior Vena Cava. N-Myc Amplification was negative, she received high risk Neuroblastoma protocol chemotherapy, but her MIBG Scan was positive after 9 courses of chemotherapy, so weekly Vinblastin, Valporic Acid, and Celecoxib was added and her chemotherapy was changed to ICE protocol. Now she has stable condition and tolerates chemotherapy very well.

\section{Case 3}

A 10 years girl known case of relapse of Astrocytoma of cerebellum, who had received full dose of radiation at the diagnosis, so she received 9 courses of Irinothecan, Avastin (Bevacizumab) based chemotherapy; but MRI showed no change in tumor size, so was put on metronomic chemotherapy (weekly Vinblastin, Valporic Acid, Celecoxib, Methotrexate and Cyclophosfamide) and now she has progression free survival in this last 6 months and had clinical improvement except for diplopia which remained persistently.

\section{Case 4}

A 3 years old boy with paraspinal mass whose mass was partially resected at the first operation diagnosed as Neuroblastoma and received Dexametasone plus 3 courses of high risk protocol chemotherapy, then was sent for second look operation in cooperation of neurosurgeon and pediatric surgeon. Again 5 courses of chemotherapy were given, but her MIBG Scan was still positive so was put on metronomic chemotherapy plus Vitamin A analogue (Racutane), and had 6 months progression free survival, laboratory and clinical improvement.

\section{Discussion}

Metronomic chemotherapy inhibits tumor progression initially through anti-angiogenesis mechanisms while prominently 
decreasing undesirable toxic adverse effects. Metronomic chemotherapy can induce tumor responses in oncology patients previously resistant to treatment or in those who have relapsed after classic chemotherapy [1]. Despite the disparity of efficacy reported for metronomic chemotherapy based on the drug combination and tumor type; all clinical trials have proven that this new treatment, alone or in combination with other treatment options, is well tolerated. [1,2] The highest grade of toxic adverse effects observed include grade 1 nausea, vomiting, grades 1 and 2 anemia, neutropenia, leukopenia and lymphopenia, as well as low-grade fatigue. A potential suggested chemotherapy regimen for Meduloblastoma/cerebral Primitive Neuro-Ectodermal Tumor (PNET), Osteosarcoma, Nephroblastoma, high grade Glioma, Hodgkin's lymphoma, Rhabdomyosarcoma, Neuroblastoma and kidney Rhabdoid tumors is listed below.

Vinblastine (3 mg/m2) weeks 1-7; Methotrexate (twice weekly, PO, $10 \mathrm{mg} / \mathrm{m}^{2}$ for 3 weeks) weeks 5-7; Celecoxib (250 mg/m2 PO, B.I.D.) daily days 1-56 or Valproic acid (20 mg/kg/day, daily); Cyclophosphamide (30 mg/m2/day, P0) days 1-21, followed by a two-week chemotherapy break [2]. This treatment protocol is scheduled for period cycles of 56 days (8 weeks). According to patients' condition and side effects; like neutropenia and mucositis; cyclophosphamide or methotrexate may be omitted in the next course. Another chemotherapeutic regimen consists of the following: The first cycle consists of weekly Vincristine $(1.5 \mathrm{mg} /$ $\mathrm{m} 2$ ) on days 1, 8, 15, and 22; daily Cyclophosphamide (25-30 mg/ $\mathrm{m} 2$ ) on days 1 to 21 ; and twice weekly Methotrexate (15 mg/m2) on days 21 to 42 , followed by a one week break. For subsequent cycles, Vincristine was administered only at weeks 1 and 5 of each cycle [2].

Inhibition of histone deacetylases (HDACs) with continuous low-dose all-trans retinoic acid (ATRA) and Valporic acid as differentiating agents has been proposed as alternative treatments in some refractory malignancies including acute Myeloblastic leukemia (AML) for prevention from tumor angiogenesis and induction of apoptosis in malignant cells [3]. Although factors that need to be optimized in metronomic chemotherapy include the number of agents to be synthesized; doses of each agent to be used, either alone or in combination; and the timing of drug administration and break therapy [4]. Currently strategies are being developed to combine metronomic chemotherapy with conventional chemotherapy administered at the maximum tolerated dose, radiotherapy, and/or targeted therapy. These strategies are opening the way for an immense number of potential combinations. Patient stratification and treatment monitoring for the best Metronomic Chemotherapy for End-stage Pediatric Oncology Patients need to be defined in the future [3,5]. In addition, a primary principal for the expansion of anti-vascular treatment, in particular metronomic chemotherapy, is based on the concept that vascular endothelial cells are genetically stable and presumably are less likely to acquire drug resistance compared to malignant cells. Evidences have suggested that tumor endothelial cells are differentiated from normal endothelial cells [6]. They have tumor-specific genetic abnormalities and acquire drug resistance. Therefore, pharmacogenetic and pharmacoproteomic studies on the tumor endothelial cells are necessary in order to recognize their sensitivity to metronomic chemotherapy which will assist in developing drugs for clinical use in the future [6].

\section{Conclusion}

Metronomic chemotherapy is an efficient intervention with tolerable side effects; for control of disease progression in most types of malignancies. It seems that it's impossible to suggest a single metronomic regimen for all types of refractory malignancies. Determining the most favorable combination regimens of metronomic chemotherapy remain a critical point for any tumor type.

\section{Acknowledgment}

None.

\section{Conflict of Interest}

No conflict of interest.

\section{References}

1. Shahriari M, Abdolkarimi B (2015) Metronomic Chemotherapy as a New Strategy for End-stage Pediatric Oncology Patients in Iran. Middle East J Cancer 6(1): 67-69.

2. André N, Abed S, Orbach D, Alla CA, Padovani L, et al. (2011) Pilot study of a pediatric metronomic 4-drug regimen. Oncotarget 2(12): 960-965.

3. Shahriari M, Abdolkarimi B, Saleh F, Salajeghe P (2015) Metronomic Maintenance Therapy in Refractory Acute Myeloblastic Leukemia with Monosomy 7. Iran J Blood Cancer (IJBC) 7(5): 231-234.

4. Fousseyni T, Diawara M, Pasquier E, André N (2011) Children treated with metronomic chemotherapy in a low-income country: MetroMali-01. J Pediatr Hematol Oncol 33(1): 31-34.

5. Jemal A, Siegel R, Ward E, Hao Y, Xu J, et al. (2009) Cancer statistics, 2009. CA Cancer J Clin 59(4): 225-249.

6. Bergers G, Hanahan D (2008) Modes of resistance to antiangiogenic therapy. Nat Rev Cancer (8): 592-603.

7. Kerbel RS (2008) Tumor angiogenesis. N Engl J Med 358(19): 20392049 